\title{
Fuentes de apoyo recibidas por las gestantes en el duelo prenatal ${ }^{1}$
}

Institución: Área de Salud de San Ramón

\section{COMO CITAR}

Araya, Z. (Abril, 2014) Fuentes de apoyo recibidas por las gestantes en el duelo prenatal. Rev. Actual de Costa Rica, 26, 1-13. Recuperado de: $<$ http://www.revenf.ucr.ac.cr/dueloprenatal.pdf> ISSN 1409-4568

Se presentan los resultados de una investigación realizada en el cantón de San Ramón, Costa Rica, con los datos del 2012 proporcionados por la Comisión Local de Análisis de Mortalidad Materno Infantil (COLAMMI). El objetivo consistió en identificar las fuentes de apoyo recibidas por las gestantes, durante su embarazo, tras enterarse de que el feto presentaba, malformaciones incompatibles con la vida extrauterina; dicho apoyo se brindó en el proceso de duelo y se pretendió conocer el impacto de ese soporte respecto de la salud mental. A partir de un enfoque cualitativo se desarrolló la metodología estudio de caso retrospectivo. La población estuvo integrada por tres mujeres gestantes, quienes participaron de forma voluntaria e informada, cuya pérdida ocurrió entre los 3 meses y el año anteriores al estudio. Se aplicó la técnica de entrevista, así como la revisión de los informes elaborados por la COLAMMI. Los resultados indican que las intervenciones recibidas por el Ier nivel de atención son escasas y muy tardías. Se concluye que el apoyo brindado por el personal de salud, en el tercer nivel de salud contribuyó a que las gestantes recibieran información acerca del duelo y llevaran el proceso de forma más favorable. En este caso, la intervención del Ier nivel de atención fue tardía y difícil de acceder.

Palabras claves: duelo prenatal, intervenciones de enfermería, atención primaria de salud

\footnotetext{
${ }^{1}$ Fecha de recepción: 12 de setiembre 2013

Fecha de aceptación: 15 enero del 2014

${ }^{2}$ Enfermera Obstetra. Área de Salud de San Ramón. Caja Costarricense de Seguro Social. Correo electrónico: zujdjp@hotmail.com
} 


\title{
Sources of support received by pregnant women in prenatal bereavement ${ }^{1}$
}

Zully Araya Cubero ${ }^{2}$

Institution: Area of Health San Ramón

\section{CITED}

\begin{abstract}
The results of research conducted in the canton of San Ramón, Costa Rica are presented, with 2012 data provided by the Local Committee on Maternal and Infant Mortality Review ( COLAMMI ). The goal was to identify sources of support received by pregnant women, during pregnancy, after learning that the fetus had, malformations incompatible with life outside the womb, this support is provided in the process of mourning and sought to know the impact of that support with regard to mental health. From a qualitative approach the retrospective case study methodology was developed. The population was composed of three pregnant women who participated voluntarily and informed, the loss occurred between 3 months and a year prior to the study. Interview technique and review of the reports prepared by the COLAMMI was applied. The results indicate that interventions received by the first level of care are scarce and very late. It is concluded that the support provided by health personnel in the third level of health helped the pregnant to receive information about the grieving process and take more favorably decision. In this case, the intervention of first level of care was delayed and was difficult to access.
\end{abstract}

Keywords: Nurse-midwife, perinatal bereavement (prenatal death), primary health care

\footnotetext{
${ }^{1}$ Date of receipt: September 12, 2013

${ }^{2}$ Nurse Midwife. Area of Heath San Ramon. CCSS. E mail: zujdjp@hotmail.com
}

Date of acceptance: January 15, 2014 


\section{INTRODUCCIÓN}

El embarazo suele ser un proceso natural en la vida de toda mujer: por lo general; es un acontecimiento que produce alegría, aunque a veces no es planeado ni aceptado; sin embargo, una vez reconocido, es un periodo en que la mujer se prepara para recibir a un nuevo ser. A pesar de lo anterior, en ocasiones se presentan acontecimientos inesperados, definidos por la Organización Mundial de la Salud (OMS) (2012) como, "anomalías congénitas, también llamadas defectos de nacimiento, trastornos congénitos o malformaciones congénitas, que ocasionan cerca de 270000 fallecimientos de recién nacidos cada año, durante los primeros 28 días de vida" ( $p$. 2), contingencia que suele enfrentar a la mujer y a su familia a un proceso de duelo prenatal.

En Costa Rica, según datos del Instituto Nacional de Estadísticas y Censo (INEC), “el grupo de malformaciones congénitas se mantiene estable entre el 2011 y el 2012, hecho que causa alrededor del 36\% de las muertes infantiles", (2013, p. 1), cifras similares a las que reporta la Comisión Local de Análisis de Mortalidad Materna Infantil (COLAMMI) de San Ramón (2013), en su informe anual, según el cual, en el quinquenio 20082012, el 35\% de las muertes infantiles ocurridas en el cantón de San Ramón sucedieron por malformaciones fetales; sin embargo, en el año 2012 los fallecimientos ocurridos en menores de un año de edad, debido a malformaciones congénitas, representaron el 50\%. (INEC, 2013, p. 2). La relevancia de la estadística anterior se debe a que sirvió para diagnosticar de manera temprana las malformaciones fetales durante la gestación, máxime que cada vez es más frecuente su detección por los avances en la tecnología.

Cuando el pronóstico de vida del feto revela incompatibilidad con la vida extrauterina, en la embarazada y en su familia se producen reacciones físicas y fisiológicas, así como reacciones emocionales como miedo, tensión y estrés ante la muerte inminente de su hijo o hija. Según lo señala López (2011), "para los progenitores no es el peso ni la edad gestacional lo que transforma el feto en hijo, sino el lugar que han creado en su corazón". (p.1). El vínculo entre la madre y el padre con sus hijos se genera desde el momento en que se toma la decisión de ser padres, entonces, nacen sentimientos de apego, así como de responsabilidad y obligaciones con el nuevo ser.

Debido a que el dolor y el proceso de duelo pueden variar de una mujer a otra según su historia de vida, así como sus implicaciones en su vida laboral, social, familiar o con la pareja, la intervención adecuada en este proceso podría evitar complicaciones mayores. Como lo indica el informe emitido por la OMS (2008), "el tratamiento de los defectos congénitos depende del nivel de asistencia sanitaria y puede ir desde la terapia médica hasta la cirugía, la rehabilitación y la atención paliativa cuando convenga" (p. 3).

La atención primaria de la salud (APS) es la base del sistema de salud costarricense y constituye el primer elemento del proceso continuo de atención en salud. La Declaración de Alma Ata, citada por la OPS (2008) definió la APS como,

atención esencial en salud basada en métodos y tecnologías prácticas, científicamente fundamentadas y socialmente aceptables, accesible a los individuos y a las familias en la comunidad, a través de su plena participación y a un costo que la comunidad y el país puedan asumir para mantenerla. (p. 6). 
Por ende, la APS debe centrarse en la persona y considerar las dimensiones física, mental, emocional, social, laboral, cultural de éstas, así como su entorno familiar y redes de apoyo.

Aparte de la APS, en Costa Rica la salud de la población está regulada por el Ministerio de Salud a través de la Ley General de Salud, la Política Nacional de Salud 2010-2021, la Política Nacional de Salud Mental, el Plan Nacional de Salud 2010-2021, entre otros instrumentos normativos que establecen la obligatoriedad de integralidad en los servicios de salud, razón por la que se considera fundamental tanto la promoción de la salud, la prevención, tratamiento y rehabilitación de la enfermedad, enfoque en el que la salud mental y la salud sexual son esenciales. Al respecto, la Caja Costarricense del Seguro Social (CCSS) (2013), como ente operativo del sistema de salud, tiene como misión "proporcionar los servicios de salud en forma integral al individuo, la familia y la comunidad y otorgar la protección económica, social y de pensiones, conforme la legislación vigente, a la población costarricense". (Plan estratégico institucional, p. 17); tal consigna incluye en la atención de salud de las personas conceptos expuestos en la definición de atención primaria en salud, planteada en párrafos anteriores.

Respecto de Enfermería, Watson (2012) menciona que,

ante el riesgo de deshumanización en el cuidado del paciente, a causa de la gran reestructuración administrativa de la mayoría de los sistemas de cuidado de salud en el mundo, se hace necesario el rescate del aspecto humano, espiritual y transpersonal, en la práctica clínica, administrativa, educativa y de investigación por parte de los profesionales de enfermería. (p.3).

Tal como se lee, la autora motiva a los profesionales en esta disciplina a reinventar su práctica e integrar el conocimiento del cuidado físico al conocimiento de la conducta humana para brindar una atención de calidad, promover la salud y ofrecer apoyo o seguimiento de sus pacientes. En cuanto al Reglamento para el Ejercicio de la Enfermería Ginecoobstétrica y Perinatal en Costa Rica (2005), establece dentro de las competencias de la profesión en la atención primaria de salud, "la detección temprana, clasificación, seguimiento y referencia de casos de riesgo y con problemas específicos y la orientación para el manejo de problemas de salud” (p.13).

Partiendo de lo mencionado y, tras analizar el orden jurídico y político en materia de salud mental y reproductiva de la población costarricense y las funciones de la Enfermería Obstétrica, surgió la inquietud de elaborar una investigación, cuyo objetivo general fue identificar la intervención en salud que reciben las mujeres, durante el proceso de duelo prenatal en la atención primaria de salud del Área de Salud de San Ramón y cómo ésta ha influido en su salud mental.

\section{MATERIALES Y MÉTODOS}

La población la integraron tres mujeres del cantón de San Ramón de Alajuela, a cuyo producto -durante su embarazo- se le diagnosticó malformaciones fetales incompatibles con la vida extrauterina; las participantes aceptaron participar voluntariamente en la investigación y la pérdida del infante ocurrió entre los 3 meses y el año anteriores al estudio.

Se desarrolló un diseño cualitativo basado en los planteamientos de Rodríguez (1996), mencionado por

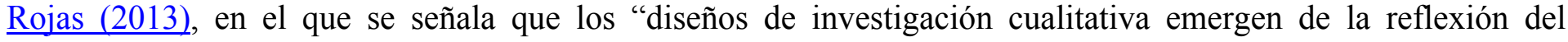


investigador, tras las primeras aproximaciones a la realidad objeto de estudio" (p.26). En ese sentido, la investigadora labora en el área de San Ramón de Alajuela, específicamente en atención primaria; por lo que su inquietud y reflexión acerca la problemática de las mujeres que enfrentan el duelo prenatal la condujo a plantear este estudio. Las fases del proceso de investigación según Rodríguez (1996) mencionado por Rojas (2009) son: 1. Preparatoria, 2. Trabajo de campo, 3. Analítica y 4. Informativa.

En la fase preparatoria, se reflexionó en cuanto al objeto de estudio y, posteriormente, se creó el diseño. Se intentó establecer un marco conceptual en el que se basa la investigación. En la etapa de diseño se planificaron las actividades que se ejecutaron posteriormente.

En la fase de trabajo de campo se procedió a recolectar la información para lo que se empleó como técnicas el estudio de caso, la entrevista y la revisión de documentos. Se ejecutó una revisión bibliográfica y de normativa institucional con el fin de obtener fundamentos para el desarrollo de la investigación, así como la definición de la metodología y la confección del instrumento para obtener la información.

Los instrumentos de recolección de datos fueron una guía de entrevista semiestructurada con 13 preguntas abiertas y una guía para la revisión de los informes elaborados por la COLAMMI.

Las entrevistas fueron grabadas y transcritas y se registraron las notas recabadas. Todos los datos obtenidos se procesaron en la matriz confeccionada para tal efecto; además, para el análisis de los datos se llevó a cabo una triangulación por técnicas, tomando en consideración las respuestas de las tres mujeres para lo que se identificó coincidencias, ideas básicas, e impacto de las intervenciones en su salud mental.

En la fase analítica se codificaron los datos de acuerdo con la unidades de análisis establecidas y las categorías. Las unidades de análisis fueron dimensión biológica, servicios de salud y duelo prenatal.

Finalmente, en la fase informativa se elaboró un informe de resultados, el cual incluye recomendaciones para las intervenciones de enfermería obstétrica en el primer nivel de atención.

\section{Consideraciones éticas}

Se consideraron los criterios de bioética contemplados en el Código de Ética y Moral Profesional del Colegio de Enfermeras y Enfermeros de Costa Rica; de igual forma, se tomó en cuenta el principio de autonomía, desde el enfoque principialista, dado que se explicó a las participantes el propósito del trabajo, se les aseguró la confidencialidad en la información brindada, se les explicó que los datos serían divulgados en una publicación científica; el principio de beneficencia se consideró para mejorar la actitud e intervenciones que brinda el o la enfermera obstetra; el de la no maleficencia, al mostrar una actitud favorable para la correcta relación con la persona. Por último, se valoró el principio de justicia, dando un trato igualitario a cada participante durante la entrevista. 


\section{RESULTADOS}

\section{Unidad de análisis: características sociodemográficas}

Las principales características sociodemográficas de los sujetos, obtenidas a partir de los informes de la COLAMMI son las siguientes: las mujeres tenían en promedio 24 años de edad; todas son costarricenses y, según su estado civil, dos convivían con su compañero en unión libre y una soltera. Respecto de la ocupación, dos de ellas eran estudiantes y una, ama de casa; su nivel educativo corresponde a secundaria completa. Las tres participantes contaban con recursos económicos para satisfacer sus necesidades básicas, dos eran aseguradas por el Estado y una por seguro directo. Dos habitaban en casa propia, mientras que una alquilaba. Sus grupos familiares poseían adecuadas relaciones interpersonales.

\section{Unidad de análisis: Dimensión biológica}

Respecto del control prenatal, las participantes mencionaron que, en un inicio, controlaron su embarazo con el equipo básico de atención integral en salud (EBAIS); aunque dos de ellas, también optaron por los servicios médicos de un especialista, así lo demuestran sus respuestas: "En el EBAIS” (partic.1); "En el EBAIS,...pero también pagué algunos controles con médico particular”. (partic.2); "Con médico ginecólogo pagado" (partic.3).

En relación con el diagnóstico y pronóstico de la vida de su hijo, las mujeres señalaron que fue dado por el médico tratante, en el EBAIS y en la consulta privada. Una participante mencionó que "en el Hospital de San Ramón me hicieron un ultrasonido y la doctora detectó algo, por lo que me envió a San José al Calderón en donde me dieron la mala noticia" (partic.1); "El ginecólogo me hizo el ultrasonido en San Carlos, pero yo quería otra opinión y me hice otro ultrasonido en la Clínica Bíblica...después llevé los exámenes al EBAIS y me refirieron al hospital de San Ramón y luego al hospital Calderón" (partic. 2); "El médico que me atendía en la consulta particular, después me refirió al Hospital de San Ramón y fui a una consulta de alto riesgo" (partic.3).

Además, las mujeres indicaron manifestaciones físicas y emocionales al momento de recibir la noticia respecto del diagnóstico y el pronóstico de vida de su hijo: "Sentí mucho miedo.... lloré... me sentía mal, muy triste (partic.1); "No lo podía creer, sentí un vacío en el estómago, ganas de vomitar ...fue muy duro pensaba en cómo iban a reaccionar mis hijos y mi familia" (partic. 2); "Me sentí culpable, porque yo no quería estar embarazada, sentí enojo hacia mi novio y con Dios.... Lloré mucho, me dolía el pecho” (partic. 3).

\section{Unidad de análisis: Servicios de salud}

En lo que respecta a la atención en crisis de primer orden al diagnóstico y pronóstico de su hijo, las entrevistadas afirmaron haber recibido algún tipo de apoyo, ellas indicaron: "El médico me tranquilizó, mi novio... la enfermera me dio agua, y después una psicóloga me estuvo hablando y dando palabras de aliento" (partic. 1); "El apoyo de mi esposo; la doctora que me realizó el examen se portó muy bien y me tranquilizó" (partic. 2); "Fue muy duro, yo estaba sola con el doctor, él me calmó y luego llamó a mi casa y mi mamá me acompaño" (partic. 3). 


\section{Revista Electrónica Enfermeria actual en costa Rica}

\section{www.revenf.ucr.ac.cr}

El apoyo individual emocional que recibieron las participantes, posterior al diagnóstico, incluyó en algunos casos la intervención del tercer nivel de atención; las entrevistadas respondieron lo siguiente: "Me refirieron al psicólogo del Hospital, también la familia y los vecinos" (partic.1); "Tuve atención psicológica en el Calderón durante el embarazo y el apoyo de mi familia" (partic. 2); "Mi mamá insistió y acudí a control con psicóloga (privada)... mi familia me apoyó en todo momento" (partic.3).

El apoyo emocional familiar -tanto de su núcleo familiar como familiares- posterior al diagnóstico fetal fue nulo. En relación con lo anterior expresaron: "No" (partic.1); "Mi esposo me acompañó a las citas, pero mis hijos sufrieron mucho" (partic. 2); "No" (partic. 3).

Por otro lado, las entrevistadas indicaron que la atención del duelo postparto, por parte del primer nivel de atención, fue tardío, difícil de acceder o inexistente, dato evidente en sus respuestas: "Todavía estoy en control, pero fue muy tardío... la cita la tuve casi dos meses después de que murió mi hijo" (partic.1); "No pude ir, porque me quedaba muy largo... pero mi esposo y yo fuimos a San Carlos a recibir terapia..." (partic. 2); "Ninguno.... Yo no quise ir al EBAIS... ni a sacar la cita con la psicóloga” (partic. 3).

Finalmente, las participantes consideran esencial el acompañamiento y el apoyo del personal de salud de atención primaria, de lo cual comentaron: "La atención que me dieron en el EBAIS fue buena, pero después que estuve en control en el hospital ya no los volví a ver... solo cuando me visitaron después de que murió mi hija, a los dos meses. Sería bueno más apoyo para uno y la familia, porque a veces no sabemos qué hacer..." (partic.1); "Creo que es importante que visiten a las personas que pasan por estos problemas... porque a veces uno se deprime mucho y no sabe con quién hablar o qué hacer." (partic. 2); "Bueno, yo no tenía seguro... y creo que sin seguro no lo atienden, el parto sí, pero la consulta con la psicóloga, no. Yo tuve algunos controles con psicólogo en la consulta privada... pero no sé cómo hacen los que no pueden pagar...” (partic. 3).

\section{Unidad de análisis: Duelo prenatal}

Las señoras recordaron que durante el embarazo vivieron todas las etapas del duelo, negación, ira, negociación, depresión y aceptación. Al respecto indicaron lo siguiente: "Fueron 10 semanas duras, casi no comía y no dormía al principio y estaba muy triste, pero después fui aceptando, la psicóloga me ayudó mucho y el apoyo de mi familia y sus oraciones me ayudaron..." (partic.1); otra manifestó que "al principio creía que tal vez se haría un milagro y oraba mucho, no tenía ganas de salir de la casa ni hacer nada en la casa, mis hijos estaban tristes, pero el apoyo y la información del médico y todo su equipo me ayudaron mucho a mí y a mi esposo, siempre fueron claros y esos nos ayudó..." (partic. 2); "A mí me lo diagnosticaron muy tarde, yo tuve 8 controles pagando y el doctor nunca vio nada... No fue hasta unos días antes de que naciera mi hija que el doctor descubrió algo en su cabeza... Me sentí enojada, triste, pero qué podía hacer" (partic. 3).

Respecto de la atención del parto, fue realizado en el nivel hospitalario, calificado en general como "buena atención y buen trato"; "Bien, yo me fui por mis propios medios al Hospital Calderón, mi novio me acompañó... Estuve tranquila, los médicos y las enfermeras fueron muy amables al final tuve parto normal" (partic.1); "Ya tenía la fecha y me comenzaron las contracciones y nos fuimos (mi esposo y yo) para el hospital, cuando llegué el corazón del bebé estaba muy bajo por lo que me hicieron cesárea. La atención fue excelente, incluso me mantuvieron internada más de lo debido para estar cerca de mi hijo..." (partic. 2); "Bueno... el doctor me 
programó cesárea, después, cuando desperté me dijeron que la bebe estaba en Pediatría porque le tuvieron que poner oxígeno y pude verla hasta en la pura tarde..." (partic. 3).

Durante el proceso de muerte del hijo, algunas tuvieron la oportunidad de estar cerca; sin embargo, este acontecimiento fue una experiencia difícil para las entrevistadas: "Yo no pude estar cuando murió, porque no me podía levantar, mi novio sí lo acompañó. Lloré mucho... y a pesar de que sabía lo que iba a pasar me deprimí mucho por lo que tuve que seguir en control con la psicóloga. El verlo en la cajita y enterrarlo fue muy difícil para mí..."(partic.1); "Cuando murió yo estaba con él, mi esposo estaba en la casa con los otros niños...fue bonito, el sacerdote oró y las enfermeras me abrazaron y me apoyaron.... Lo difícil fue llegar a la casa y explicarle a los niños lo que pasaba" (partic. 2); "El proceso fue muy largo y difícil... primero me dijeron que no pasaba de un mes y duró siete, después me dijeron que no se movería ni haría nada y ella me veía y yo sentía que se alegraba, cuando me veía llegar, después me dijeron que nunca comería, porque no podía tragar pero yo le di fruta, después se complicó y la internaron en el Hospital de Niños, donde murió... yo tenía la esperanza de que viviera más, porque ella había hecho todo lo que me decían que no haría y después se fue..." (partic. 3).

En cuanto al duelo anticipado durante el proceso de gestación, las participantes consideraron muy valiosa la ayuda recibida por parte del personal de salud, dado que les ayudó a sobrellevar la pena y el dolor. Al respecto afirmaron: "Sí, claro... eso me dio fortaleza... pero necesité más ayuda" (partic.1); "Sí, porque me atendieron bien y me aclaraban mis dudas, siempre me explicaron todo muy claro en el hospital. También mi familia y la iglesia me ayudó" (partic. 2); "La verdad es que yo no quería hablar del problema con nadie ni con mi familia, ni el papá de la bebé... estaba muy adolorida y sentía mucha culpa... tal vez si hubiera hablado me hubiera sentido más aliviada... (partic. 3).

Durante el postparto, las entrevistadas comenzaron nuevamente las etapas de duelo, aunque ya más conscientes de la realidad, a lo que respondieron: "Estuve triste, deprimida, me da miedo estar sola, casi no comía" (partic.1); "He tenido que estar fuerte por mis hijos y mi esposo, pero no me he sentido nada bien, lloro a escondidas o cuando no están" (partic. 2); "El trabajo me hace bien porque me entretengo y a ratos se me olvida, el problema es cuando llego a mi casa y entro al cuarto y veo sus cosas: me pongo muy mal..."(partic. 3).

Las principales fuentes de apoyo recibidas por las gestantes durante el proceso de duelo fueron brindadas por el tercer nivel de atención, así como por sus familiares cercanos, lo cual impactó en su salud mental, dado que les ayudó a sobrellevar la pena y el dolor y les permitió vivir de manera más serena la experiencia del embarazo, parto y muerte de su hijo (a). A pesar de ser una experiencia difícil para las entrevistadas, la madre que recibió poco apoyo emocional fue la que más sufrió la pérdida.

En lo que concierne a la atención del duelo postparto, por parte del primer nivel de atención, fue tardío, difícil de acceder o nulo; por ende, es necesaria una reflexión tanto para el sistema de salud, como para los profesionales que laboran en ese nivel. 


\section{DISCUSIÓN}

El dolor que causa la posibilidad de perder un hijo produce diversas manifestaciones físicas, emocionales y espirituales en los padres y familiares cercanos, las cuales pueden perdurar varios meses o, incluso, años. En ocasiones, el duelo mal sobrellevado puede convertirse en estrés, depresión o algún otro trastorno psicológico grave, motivo por el que, durante esta dolorosa experiencia, la gestante y su familia deben recibir apoyo del personal de salud capacitado, que se encargue de aclararles sus dudas, favorecer la comunicación, brindar contención y acompañamiento cuando se requiera.

\section{Según López (2011),}

el duelo perinatal tiene unas características que lo diferencian de duelos de otros tipos: la proximidad entre el nacimiento y la muerte, la juventud de los progenitores para los que supone su primer contacto con la muerte y lo inesperado del suceso. Hay que añadir que se trata de la pérdida de una relación más simbólica que real, basada en sus necesidades y deseos; sin embargo para los futuros padres comprender el panorama que se avecina es un factor protector de su calidad de vida personal y de pareja, así como un medio para que obtengan herramientas para afrontar la muerte (p.62).

La Conferencia Internacional sobre la Población y el Desarrollo (1994), vigente y ratificada por Costa Rica en el año 2000, indica que toda persona tiene derecho al disfrute más alto de salud física y mental; en el capítulo VII define la salud reproductiva como "un estado general de bienestar físico, mental y social, y no de mera ausencia de enfermedades o dolencias, en todos los aspectos relacionados con el sistema reproductivo y sus funciones y procesos" (p. 37). Si se entiende la salud sexual y reproductiva como componente esencial de la salud, es fundamental disponer de servicios accesibles brindados por el primer nivel de atención en caso de duelo perinatal; la Conferencia señala, además, la interrupción del embarazo como una opción válida, de conformidad con la legislación de cada país.

El Código Penal de Costa Rica, en su artículo 21 señala que,

No es punible el aborto practicado con consentimiento de la mujer por un médico o por una obstétrica autorizada, cuando no hubiere sido posible la intervención del primero, si se ha hecho con el fin de evitar un peligro para la vida o la salud de la madre y éste no ha podido ser evitado por otros medios (1971).

Los diagnósticos prenatales de malformaciones congénitas letales en Costa Rica van acompañados de la imposibilidad de interrumpir el embarazo, curar o rehabilitar al feto; no obstante, debe abordarse y paliar el dolor de la gestante y su familia. 


\section{Revista Electrónica Eneremeria actual en costa Rica}

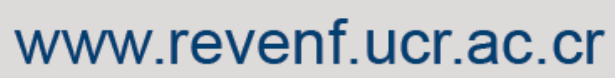

La Organización Panamericana de la Salud (2008), en su informe "La Atención primaria de la salud más necesaria que nunca”, en su capítulo 3 apunta que la persona es prioritaria y describe características esenciales para conseguir mejores resultados sociales y de salud,

las cuales son la centralidad de la persona, la integralidad y la integración, y la continuidad de la asistencia, con un punto de acceso sistemático al sistema sanitario para que los pacientes y quienes les atienden puedan entablar una relación duradera basada en la confianza, incluyendo la capacidad de coordinar el apoyo brindado por los hospitales, los servicios especializados y las organizaciones de la sociedad civil (p. 44).

Si bien es cierto las entrevistadas recibieron atención emocional, esta correspondió solo al tercer nivel de atención. El programa de atención primaria de la salud en Costa Rica y las Normas de Atención integral en salud, primer nivel de atención, actualmente prestan poca atención a los procesos de duelo, vividos por las gestantes y su familia. Según la Política Nacional de Salud mental 2012-2021, hay una pobre capacidad instalada y escaso recurso humano capacitado o especializado, "asimismo, no existen políticas de formación de recurso humano para atender las necesidades actuales y futuras en el campo de la salud mental” (p.26).

El papel que desempeña la atención primaria de salud y en especial el profesional de enfermería es limitado en el ámbito de la salud mental, lo cual impide la capacidad resolutiva en el contexto comunitario. Según el informe regional sobre los sistemas de salud mental en América Latina y el Caribe (Who-aims. 2013),

la capacitación/o formación (de pregrado y postgrado) en temas de salud mental que recibe el personal de APS (profesionales médicos, enfermeros/as, entre otros) es escasa. Como consecuencia, la capacidad resolutiva es reducida y, en términos generales y con las debidas excepciones, no existen mecanismos consolidados, sistemáticos y viables de referencia $\mathrm{y}$ contrarreferencia (p. 64).

El informe Who-aims (2013) señala la falta de recurso humano, el cual se concentra en los hospitales psiquiátricos "dejando poca disponibilidad para el desarrollo de servicios en la comunidad. En algunos casos, el grado de capacitación y responsabilidad otorgado al personal de enfermería ha podido suplir, de manera altamente positiva, la falta inicial de personal médico" (p. 64).

La falta de enfermeras obstetras en atención primaria es una limitante; sin embargo en las áreas de salud donde exista el recurso, le corresponde trabajar los derechos sexuales y reproductivos en situaciones especiales y asesorar tanto a la gestante, su familia y la comunidad, en el duelo por aborto, duelo prenatal o duelo por muerte perinatal o neonatal, circunstancias en donde se enfatizan las necesidades cognitivas, socioculturales, económicas y espirituales de la población en su contexto. En este sentido, la actitud de la práctica obstétrica debe estar dirigida hacia la actualización y el trabajo orientado a la enfermería basada en la evidencia, como indica Watson en su teoría de enfermería sobre el cuidado humano.

Concejo et al (2002) proponen un "programa de salud para la atención del duelo en atención primaria" (p. 1), con el fin de proporcionar a la mujer y su pareja los instrumentos necesarios para superar favorablemente el 
proceso de duelo ante la pérdida del hijo; al respecto, la enfermería obstétrica en el primer nivel de atención podría incluir esta tarea en conjunto con el equipo interdisciplinario de salud.

La guía para los profesionales de la salud ante situaciones de duelo (2011) motiva al abordaje de los procesos de duelo desde un modelo de promoción-prevención visto no como una enfermedad, sino como un proceso de elaboración de una pérdida en el que no se fuerza el duelo, con el fin de que sea de una manera determinada, puesto que algunos dolientes requerirán servicios de salud que incluirán "actuaciones puntuales (en una única sesión) o una actuación más continuada (seguimiento)" (p. 31). Dicha guía no recomienda ninguna intervención específica en el duelo normal, pero sí menciona la necesidad de contar con "herramientas para identificar claramente y de forma precoz un duelo con riesgo de convertirse en patológico" (p 31).

Flenady y Wilson (2008) sostienen que "no hay suficiente información acerca de los tipos de intervenciones y sus posibles beneficios para brindar apoyo a las madres, padres y sus familias, después de la muerte de un recién nacido" (p.1). La revisión indicó que son necesarios más ensayos, metodológicamente rigurosos; aunque sí existe suficiente información sobre las consecuencias de un duelo mal elaborado.

De acuerdo con la Guía para la atención a la muerte perinatal y neonatal, citando a Keller (2009), "la muerte de un hijo es la pérdida más profunda que una persona puede experimentar. Como sociedad, estamos más preparados para lidiar con otras muertes... esto causa profundos sentimientos de desorientación que ningún otro tipo de pérdida" (p.2).

Algunas intervenciones de enfermería que se podría ejecutar para guiar a la madre y familia serían:

- Visitar el domicilio de la gestante para un primer acercamiento y valoración del riesgo y propiciar un ambiente de cordialidad y familiaridad con el Ier nivel de atención.

- Dar contención y apoyo emocional del "duelo prenatal" anticipado, si se requiere.

- Facilitar la expresión de los miedos por los defectos físicos del feto.

- Apoyar y orientar el duelo con familiares, amigos y la comunidad en general

- Brindar acompañamiento con calidad y calidez en todo su proceso de pérdida perinatal.

- Recomendar en el postparto, la donación de la leche materna y no solo evitar el proceso normal de la bajada de la leche.

- Establecer programas de continuidad y grupos de apoyo comunal de esta población.

- Capacitar al personal de enfermería para el abordaje de estas pacientes.

También se evidencia la necesidad de crear protocolos y guías de actuación para orientar a las y los profesionales en enfermería obstétrica en el abordaje de las situaciones de muerte fetal en los diversos contextos (prenatal, parto o post parto), en los tres niveles de atención.

\section{CONCLUSIÓN}

El apoyo brindado por el personal de salud en el Tercer Nivel de Salud contribuyó a que las gestantes se informaran sobre el proceso y vivieran el duelo de forma más favorable; sin embargo, la intervención del Primer 


\section{Revista Electrónica Enfermería Actual en costa Rica}

Nivel de atención fue tardío y difícil de acceder; por tanto, es imprescindible crear acciones desde este nivel para solventar las carencias en este tipo de casos.

\section{REFERENCIAS BIBLIOGRÁFICAS}

Área de Salud de San Ramón (2013). Informe Anual de la COLAMMI 2012. San Ramón, Costa Rica.

Caja Costarricense de Seguro Social (2013). Plan estratégico institucional reajustado macropolíticas 2013-2016.

Caja Costarricense de Seguro Social y Ministerio de Salud (1995) Normas de Atención integral en salud, Primer Nivel de Atención. Costa Rica.

Código Penal (1971). Ley 4573. Costa Rica.

Colegio de enfermeras y enfermeros de Costa Rica (CEYECR) (2009). Código de ética y moral de trabajo. Gaceta 18. Costa Rica.

Colegio de enfermeras y enfermeros de Costa Rica. (2005) Reglamento de Enfermería Ginecoobstetra y Perinatal de Costa Rica. Gaceta 10. Costa Rica.

Concejo, S., del Álamo, G., Martín, I., Martín, E., Poza, L., Romero, B., Saiz, S., Fernández, M. y Arroyo, F. (2002). Programa de Salud en atención primaria para la atención del duelo por la pérdida de un hijo en el periodo Perinatal. Recuperado desde http://www.federacion-matronas.org/revista

Flenady, V. y Wilson, T. (2008). Apoyo a madres, padres y familias después de la muerte perinatal. (Revision Cochrane traducida). En Biblioteca Cochrane Plus 2009 Número 3. Oxford: Update Software Ltd. Disponible en: http://www.update-software.com

Informe de la Conferencia Internacional sobre la Población y el Desarrollo (1994). El Cairo. Recuperado en: http://daccess-dds-ny.un.org/doc/UNDOC

Instituto Nacional de Estadística y Censos (INEC) (2013). Noticias: descendió la mortalidad infantil. Recuperado desde http://www.inec.go.cr/Web/Home/Noticia.aspx?id=12

Ley general de salud (1973). Ley $N^{\circ}$. 5395., Costa Rica: Gaceta N. 222, noviembre 1973.

López, A. (2011). Duelo Perinatal: Un secreto dentro de un misterio. Recuperado en http://scielo.isciii.es/pdf/neuropsiq/v31n1/05.pdf

Ministerio de Salud (2012). Política Nacional de Salud mental 2012-2021. Costa Rica.

Ministerio de Salud (2010). Política Nacional de Salud, 2010-2021. Costa Rica. 


\section{Revista Electrónica Enfermería Actual en costa Rica}

Organización Mundial de la Salud (2008). Informe sobre la salud en el mundo .La atención primaria de salud, más necesaria que nunca.

Organización Mundial de la Salud (2012). Anomalías congénitas Nota descriptiva $N^{\circ} .370$, Octubre de 2012 recuperado desde http://www.who.int/mediacentre/factsheets/fs370/es/

Organización Panamericana de la Salud (2008). Sistemas de salud basados en la Atención Primaria de Salud: Estrategias para el desarrollo de los equipos de APS. Washington, D.C.: OPS.

Rojas, L.(2009) Elementos conceptuales y metodológicos de la Investigación Cualitativa. San José: Universidad de Costa Rica.

(s.a.) (2011). Guía para los profesionales de la salud ante situaciones de duelo Recuperado desde http://testamentovital.wikispaces.com/file/view

(s.a.) (2009) Guía para la atención a la muerte perinatal y neonatal, Anexo I-V. En http://www.umamanita.es/Contenidos/Profesionales/Guia\%20Combined.pdf

Watson, J. (2012). El Cuidado. Recuperado desde http://teoriasdeenfermeriauns.blogspot.com/2012/06/jeanwatson.html

Who-aims (2013). Informe regional sobre los sistemas de salud mental en América Latina y el Caribe.

Recuperado desde http://www.paho.org/per/images/stories/FtPage/2013/WHO-AIMS.pdf 